\title{
Vídeo como ferramenta remota de educação em saúde: ações extensionistas interprofissionais durante a pandemia
}

Video as a remote tool for health education: interprofessional extensionist actions during the pandemic

Video como herramienta remota de educación en salud: acciones extensionistas interprofesionales durante la pandemia

\begin{abstract}
Resumo
O presente trabalho relata a experiência durante a disciplina optativa "Ações de promoção de saúde em Odontologia" da Universidade Franciscana de Santa Maria - RS. Através da disciplina, foram planejadas ações remotas devido à pandemia do COVID-19. Atendendo à demanda levantada por um Centro Terapêutico (CT), estudantes de Odontologia e Psicologia planejaram ações interprofissionais a fim de promover conhecimento e novos hábitos de saúde. Com o objetivo de manter o vínculo com os usuários, apesar do distanciamento social da pandemia, foi criado um vídeo explicativo sobre os cuidados em saúde bucal e, posteriormente, foram analisadas suas correlações com a saúde mental dos usuários. Como resultado da interprofissionalidade, surgiu a autonomia no cuidado da saúde e formação de hábitos desta população. Conclui-se que, apesar da distância, a união dos alunos por meio do conhecimento compartilhado permitiu criar hábitos em saúde, mantendo o vínculo e o contato com a comunidade, respeitando o distanciamento social.
\end{abstract}

Palavras-chave: Autocuidado; Pandemias; Odontologia; Psicologia; Educação em saúde.

\begin{abstract}
This work reports the experience, during the elective discipline "Ações de promoção de saúde em Odontologia" of the Franciscan University of Santa Maria - RS. Through the discipline, remote actions were planned due to the COVID-19 pandemic. Meeting the demand raised by a Therapeutic Center (Centro Terapêutico - CT), students of Dentistry and Psychology, planned interprofessional actions to promote knowledge and new health habits. In order to maintain the link with users, despite the social distance from the pandemic, an explanatory video on oral health care was created and subsequently analyzed its correlations with the mental health of users. As a result of interprofessionality, autonomy in health care and formation of habits of this population emerged. It is concluded that despite the distance, the union of the students through shared knowledge allowed to create habits in health, maintaining the bond and contact with the community, respecting social distance.
\end{abstract}

Keywords: Self-care; Pandemics; Dentistry; Psychology; Health education.

\section{Resumen}

El presente trabajo relata la experiencia, durante la disciplina optativa "Ações de promoção de saúde em Odontologia" de la Universidad Franciscana de Santa Maria - RS. A través de la disciplina, se planearon acciones remotas debido a la pandemia de COVID-19. Atendiendo la demanda planteada por un Centro Terapéutico (CT), estudiantes de Odontología y Psicología, planificaron acciones interprofesionales, para promover conocimiento y nuevos hábitos de salud. Con el objetivo de mantener el vínculo con los usuarios, a pesar del distanciamiento social de la pandemia, se creó un video explicativo sobre atención en salud bucal y posteriormente analizado sus correlaciones con la salud mental de los usuarios. Como resultado de la interprofesionalidad, surgió la autonomía en el cuidado de la salud y formación de hábitos de de esta población. Se concluye que a pesar de la distancia, la unión de los alumnos por medio del 
conocimiento compartido permitió crear hábitos en salud, manteniendo el vínculo y el contacto con la comunidad, respetando el distanciamiento social.

Palabras clave: Autocuidado; Pandemias; Odontología; Psicología; Educación en salud.

\section{Introdução}

O uso de drogas lícitas ou ilícitas por parte do sujeito pode, com o passar do tempo, afetar suas relações pessoais e gerar consequências para a sua saúde. Algumas das consequências pode ser a diminuição do contato com seus familiares e a dependência química (Lopes, Silva, Santos, \& Oliveira, 2019). O usuário ou usuária muitas vezes recorre aos serviços de saúde para realizar tratamentos ou intervenções. Nesses casos, encontram-se disponíveis os serviços dos Centros de Atenção Psicossocial Álcool e Drogas (CAPS AD), pertencentes ao Sistema Único de Saúde (SUS), e as comunidades terapêuticas (CTs) (Fossi \& Guareschi, 2015).

Em relação às CTs, as mesmas tiveram seu início em 1950, na Inglaterra. As CTs obtiveram o apoio dos militares a fim de tratar os reflexos da guerra e por serem uma alternativa aos hospitais psiquiátricos. Nelas são desenvolvidas atividades educativas, laborativas e a convivência em grupo (Santos, 2018). Atualmente, encontra-se essa modalidade de atendimento como auxílio na reinserção social, oferecendo a oportunidade de mudança nas escolhas pessoais do sujeito, adquirindo novas virtudes em um local estruturado e com profissionais à disposição (Fossi \& Guareschi, 2015).

Como forma de conhecer a realidade de uma comunidade e abranger o contato com a população, as ações extensionistas são utilizadas como método de ensino com alunos da graduação. As mesmas permitem buscar soluções com um mesmo objetivo, a partir da demanda de cada local e comunidade (Ferraz \& Del Ciampo, 2019).

Ademais, com a atuação interdisciplinar, busca-se ampliar a visão perante determinadas situações. A interdisciplinaridade possibilita a colaboração dos membros e a construção de conhecimento em prol da promoção de saúde. A partir dessa abordagem, profissionais da área da saúde atuam promovendo ações para disseminar o conhecimento à população, possibilitando a formação de novos hábitos, autonomia e conscientização através da educação em saúde (Rios, Sousa, \& Caputo, 2019).

A dependência química que mais afeta a população atualmente é o uso do tabaco, ocasionando cerca de 3 milhões de mortes por ano. O consumo de tabaco e a exposição à sua fumaça (fumo passivo) são as principais causas de doenças cardiovasculares. Apesar disso, pesquisas revelam que a população pouco compreende os múltiplos perigos de saúde advindos do cigarro (OPAS Brasil, 2019).

Além disso, diante do atual cenário do coronavírus, fumantes são mais vulneráveis à infecção por Covid-19 devido ao aumento do risco de adoecer as vias aéreas e a probabilidade de possuir ou contrair doenças respiratórias, como asma, enfisema pulmonar, bronquite crônica e doença pulmonar obstrutiva (DPOC). O contato com a boca, através do ato de fumar, também facilita a contaminação, aumentando a possibilidade de transmissão do vírus (Saúde Brasil, 2020).

Com isso, o objetivo se dá por relatar a experiência de estudantes do curso de Odontologia junto à estudantes do curso de Psicologia, bolsistas do PET-Saúde Interprofissionalidade, em um CT, promovendo ações de promoção de saúde.

\section{Metodologia}

Trata-se de um relato de experiência acerca de uma atividade realizada na disciplina optativa de "Ações de Promoção de Saúde em Odontologia", sobre a atuação em um Centro Terapêutico, nos meses de março a junho de 2020, na cidade de Santa Maria-RS.

O relato de experiência é definido como uma descrição da vivência profissional que pode ser de um ou mais autores, sendo importante para o ambiente estudado, auxiliando na discussão, análise e proposição de recursos que visam a melhoria da assistência e cuidado em saúde (Flick, 2004). 
Utilizou-se uma metodologia ativa, tendo como método o Arco de Maguerez, apresentado pela primeira vez por Bordenave e Pereira, em 1982, na elaboração e realização das atividades (Reibnitz \& Prado, 2006), de forma presencial e a distância, devido à pandemia de Covid-19, a qual acarretou no distanciamento social. Sendo assim, a atividade foi readaptada para que sua execução fosse possível.

As ações foram pensadas, planejadas e executadas interprofissionalmente, através do trabalho conjunto entre Odontologia e Psicologia, visando atender as necessidades dos usuários de forma integral e efetiva por meio da prevenção e promoção de saúde.

Para isso, os estudantes de Odontologia construíram um vídeo explicativo sobre saúde bucal com o objetivo de realizar uma forma de educação em saúde de modo remoto para os usuários do CT. Junto a isso, possibilitou uma forma de estar presente, apesar da distância presencial.

O vídeo foi publicado no YouTube e, posteriormente, o link foi enviado para os usuários do CT, para que pudessem visualizá-lo. Após a visualização do vídeo pelos participantes, foi enviado um retorno através de fotos e vídeos dos usuários assistindo ao vídeo e agradecendo o contato e os kits de higiene (escova dental, creme dental, fio dental e sabonete) recebidos, entregues no CT para utilização após o aprendizado.

O encerramento da atividade foi realizado através de uma videochamada pela plataforma Zoom. Junto aos usuários do CT, foi recebido o retorno sobre o vídeo e feita a análise de como foi a compreensão dos mesmos sobre os conteúdos elaborados pelos estudantes, sendo uma oportunidade de esclarecer dúvidas, além de permitir o estreitamento do vínculo.

\section{Resultados e Discussão}

Anterior ao início das atividades, foram ministradas aulas teóricas com o estudo do Arco de Maguerez. Junto a isso, foram disponibilizadas maiores informações acerca do CT, o qual seria o local de atuação.

Após, realizou-se uma visita ao CT para reconhecimento da realidade do local e de seus usuários. Os estudantes procuraram observar fatos e acontecimentos que pudessem gerar dúvidas e/ou dificuldades.

No primeiro encontro com os usuários, os estudantes puderam conhecer cada um dos integrantes do CT, e os mesmos foram apresentados a cada estudante. Através disso, foi iniciada a formação do vínculo, atuando de forma cordial, com uma escuta qualificada das expectativas, dúvidas e vivências, respeitando o tempo de cada um, bem como a vontade em sua participação (Wolf, 2002).

Através do diálogo com os usuários, foram elencados temas sobre saúde a serem elaborados em atividades para aplicar neste contexto. Os estudantes da Odontologia e Psicologia compartilharam seus conhecimentos para o planejamento e execução de futuras atividades com os usuários.

As temáticas levantadas foram estudadas previamente pelos estudantes. Através de aulas por videochamadas e fóruns de atividades, os estudantes foram se capacitando sobre os conteúdos necessários ao planejamento das atividades. Diante disso, com os avanços alcançados, surgiram hipóteses de soluções em forma de atividades a serem aplicadas.

Dentre os temas especificados, surge o seguinte desafio: como manter o vínculo durante o isolamento social e realizar ações de educação em saúde? Reunidos em duplas, os estudantes realizaram a gravação de vídeos curtos, abordando temáticas em saúde. Após, esses vídeos foram reunidos e se tornaram um único vídeo, sendo enviado, então, ao CT.

Os vídeos continham informações de que os estudantes estavam em segurança, elaborando atividades pensadas exclusivamente aos usuários. Ademais, haviam orientações sobre a lavagem de mãos, uma temática essencial em virtude da pandemia do COVID-19.

Foram transmitidos cuidados com a higiene bucal, como a utilização do fio dental, que consiste em deslizar o fio entre os espaços dentários, na região lateral dos dentes, incluindo a face aderida por gengiva. Também a técnica de escovação, 
utilizando a limpeza mecânica dos elementos dentários. Esses cuidados foram abordados, prezando a criação do hábito no cuidado (Barros, 2007).

Dúvidas e curiosidades sobre o tema foram solucionadas, como, por exemplo:

a) Pode ser utilizado o fio dental quando a gengiva sangra?

b) Goma de mascar faz mal para os dentes?

c) Dor ao tomar algo gelado é normal?

d) Uma boa escovação deve ser demorada?

e) A higienização noturna é mais importante?

f) Boa higiene bucal depende da quantidade de creme dental?

Após assistir ao vídeo, cada membro da comunidade utilizou seu kit de higiene com os ensinamentos adquiridos. Como retorno aos estudantes, fotos e vídeos foram enviados com agradecimentos.

Em relação à Psicologia, observou-se que o vídeo é uma ótima alternativa para estimular a autonomia dos sujeitos. Através das explicações atenciosas por parte dos estudantes de Odontologia, os usuários obtiveram instruções sobre os aspectos abordados em vídeo e, com as novas informações, puderam colocar em prática os conhecimentos adquiridos, praticando e percebendo a importância de cuidar de sua saúde.

Por se tratar de um grupo de fumantes, junto aos riscos psicossociais atrelados ao consumo de tabaco, a educação em saúde acerca do uso da substância somado à higienização adequada de boca e mãos também se mostrou importante devido ao contexto da pandemia, visto que os usuários são considerados pertencentes ao grupo de risco pelo contato constante das mãos na boca e região do rosto, além das consequências ao aparelho respiratório, causadas pelo cigarro.

Caso o fumante leve as mãos não higienizadas à boca, pode contrair o vírus. Tabagistas têm o sistema respiratório prejudicado pelo fumo e, portanto, podem desenvolver a forma mais severa do coronavírus (Saúde Brasil, 2020).

Em relação à Odontologia, observou-se a criação de novos hábitos através da autonomia adquirida com o vídeo. Também, a introdução desse conhecimento com a distância, resultando em reflexos no cuidado, na saúde de forma geral e na saúde bucal.

O uso de drogas lícitas ou ilícitas propicia o surgimento ou agravo de doenças bucais, principalmente associadas à falta de cuidado com a higiene bucal. Dentre elas, pode ocorrer a diminuição do fluxo salivar, caracterizado como xerostomia, também alterações inflamatórias na gengiva e alterações celulares, como câncer bucal (Marques et al., 2015). Para prevenir esses malefícios, através da orientação, motivação e promoção em saúde, é possível manter a saúde bucal, atrelada com o controle do uso de substâncias psicoativas.

Em relação ao trabalho interprofissional, a Educação Interprofissional em Saúde (EIP) demonstra, a cada dia, ser essencial para o cuidado integral ao sujeito, possibilitando a efetividade do cuidado em saúde (Peduzzi, Silva, \& Leonello, 2018).

A EIP visa promover o trabalho conjunto entre estudantes de diferentes cursos de graduação em saúde. Se reconhece, na proposta da EIP, a relação recíproca da mútua influência entre educação e atenção à saúde (Peduzzi, 2016).

Sobre a definição de educação interprofissional, Barr (2002) relata que: "A educação interprofissional ocorre quando duas ou mais profissões aprendem entre si, com e sobre as outras, para melhorar a colaboração e a qualidade dos cuidados" (p. 2).

Em vista disso, a oportunidade de trabalhar interprofissionalmente entre Odontologia e Psicologia mostrou-se uma importante experiência. Através do conhecimento compartilhado e maior conhecimento sobre a área de cada um, permite-se a formação de um profissional em saúde especializado para as diversas demandas emergentes dos sujeitos e suas questões em relação à saúde.

O trabalho interprofissional entre Odontologia e Psicologia, em alguns momentos, é desafiador, devido à especificidade 
das áreas. Entretanto, através da comunicação efetiva do grupo e o interesse no aprender, a interprofissionalidade se concretizou, tendo como resultado a satisfação dos usuários através dos retornos positivos recebidos, possibilitando uma melhor qualidade de vida através de um maior conhecimento sobre saúde mental e bucal.

\section{Conclusão}

Observou-se a obtenção de novos hábitos por parte dos usuários após o recebimento do vídeo enviado pelos estudantes e o consumo de seu conteúdo. Por meio dos conhecimentos divulgados no vídeo, a autonomia foi desenvolvida e reforçada através da educação em saúde, permitindo, assim, o cuidado em saúde por parte do próprio sujeito, o qual desenvolveu a importância de cuidar da sua própria saúde através de novos hábitos adquiridos.

Apesar do distanciamento social, o vínculo estreito entre estudantes e usuários foi mantido. Mesmo com as adaptações necessárias, trabalhou-se para manter o contato mais humano, uma escuta qualificada e a empatia neste período tão sensível para todos.

Através da experiência relatada, foi demonstrada a importância do trabalho interprofissional entre estudantes do curso de Odontologia e Psicologia. O compartilhar de conhecimentos de cada área permite a ampliação de saberes, atuando de forma integral no atendimento à população assistida (usuários do CT), contribuindo para a formação do futuro profissional em saúde.

Ao final desta pesquisa, percebe-se a necessidade de mais estudos sobre a prevenção e promoção de saúde, autonomia e autocuidado através da prática interprofissional, a qual contribui para o cuidado da população assistida, visto que abarca as diversas variáveis dos sujeitos, além da capacidade de adaptação de diversas situações no cuidado em saúde. Junto a isso, notase a escassez de produções científicas sobre trabalhos interprofissionais entre Psicologia e Odontologia, o qual demonstra o pioneirismo do trabalho realizado e a importância de fortalecer mais trabalhos como este, visto a riqueza da atuação para ambas as áreas e para as pessoas assistidas.

\section{Referências}

Barr, H. (2002). Interprofissional Education: today, yesterday and tomorrow. United Kingdom: Centre for the Advancement of Interprofessional Education.

Barros, C. M. S. (coord.). (2007). Manual técnico de educação em saúde bucal. Rio de Janeiro: SESC, Departamento Nacional.

Ferraz, I. S., \& Del Ciampo, L. A. (2019). Uma experiência exitosa de meio século da Universidade de São Paulo junto à comunidade. Medicina, 52 (1), 1-6.

Flick, U. (2004). Uma introdução à pesquisa qualitativa (2a ed.). Bookman.

Fossi, L. B., \& Guareschi, N. M. F. (2015). O modelo de tratamento das comunidades terapêuticas: práticas confessionais na conformação dos sujeitos. Estudos e Pesquisas em Psicologia, 15 (1), 94-115.

Lopes, L. L. T., Silva, M. R. S., Santos, A. M., \& Oliveira, J. F. (2019). Ações da equipe multiprofissional do Centro de Atenção Psicossocial Álcool e Drogas. Revista Brasileira de Enfermagem, 72 (6), 1624-1631.

Marques, L. A. R. V., Lotif, M. A. L., Rodrigues Neto, E. M., Dantas, T. S., Soares, J. L., Melo, J. A., \& Lobo, P. L. D. (2015). Abuso de drogas e suas consequências na saúde oral: uma revisão de literatura. Arquivo Brasileiro de Odontologia, 11(1), 26-31.

OPAS Brasil. (2019). Folha informativa - Tabaco. https://www.paho.org/bra/index.php?option=com_content\&view=article\&id=5641:folha-informativatabaco\&Itemid=1097

Peduzzi, M. (2016). O SUS é interprofissional. Interface - Comunicação, Saúde, Educação, 20 (56), $199-201$.

Peduzzi, M., Silva, J. A. M., \& Leonello, V. M. (2018). A formação dos profissionais de saúde para a integralidade do cuidado e a prática interprofissional. Santo André: UFABC.

Reibnitz, K. S., \& Prado, M. L. (2006). Inovação e educação em enfermagem. Cidade Futura.

Rios, D. R. S., Sousa, D. A. B., \& Caputo, M. C. (2019). Diálogos interprofissionais e interdisciplinares na prática extensionista: o caminho para a inserção do conceito ampliado de saúde na formação acadêmica. Interface-Comunicação, Saúde, Educação, 23, e180080.

Santos, M. P. G. (2018). Comunidades terapêuticas: temas para reflexão. IPEA. 
Research, Society and Development, v. 10, n. 3, e38910313474, 2021

(CC BY 4.0) | ISSN 2525-3409 | DOI: http://dx.doi.org/10.33448/rsd-v10i3.13474

Saúde Brasil. (2020). Coronavírus e tabagismo: por que essa relação não dá certo? https://saudebrasil.saude.gov.br/eu-quero-parar-de-fumar/coronavirus-etabagismo-por-que-essa-relacao-nao-da-certo

Wolf, S. (2002). Psicologia no consultório odontológico. Arte \& ciência 